\title{
Design and maintenance method of a kind of rehabilitation fitness machine
}

\author{
Yan Chen ${ }^{1, a}$, Zhaoxia Liu ${ }^{1, *}$ \\ ${ }^{1}$ College of Mechanical and Electronic Engineering, Wuhan Donghu University, Wuhan, China
}

\begin{abstract}
With the rapidly increase of people's living standard, more and more people hope to get healthier and longer life. In this study, a kind of rehabilitation fitness machine is designed for disabled people with lower limb, and its daily maintenance work is clearly displayed. The design of the new rehabilitation exercise machine is suitable for disabled people to exercise, which is designed with the theory of ergonomics. Based on ergonomics theory, the design concept of the space, new structure, working space and display device of the six-link mechanism satisfies the conditions of strength and stability.
\end{abstract}

\section{Introduction}

Recently, a great number of urban and rural residents are no longer satisfy the lifestyle of food, clothing and three meals a day, and they incline to pay attention to the quality of life. Therefore, more people hope to have a healthy body to study, work better and prolong life. In recent ten years, fitness equipment products in the market have changed rapidly and steadily, especially since 1997 , the National Sports Commission actively advocates and organizes the "National Fitness Project". At present, new fitness equipment, including outdoor fitness equipment and fitness path products, achieved the rapid development, which had greatly promoted the development of national fitness.

In some developed countries and regions of the world, such as the United States, Germany, Australia, South Korea, Singapore, Taiwan, and Hong Kong, the consumer and market turnover of fitness equipment had grown rapidly in the past decade. Basing on data from the American Sporting Goods Manufacturers Association (SGMA) since 1990, annual sales of fitness equipment have been about $\$ 3$ billion to $\$ 3.5$ billion, accounting for about $30 \%$ of the national sportswear turnover. However, compared with developed countries, the development of the fitness equipment industry in China is only an immature market, which will face great obstacles to the progress of the next decade. Until 2015, the first batch of well-known brands in China's fitness equipment industry: Outlet, Haoshijia, Shuhua, Wannianqing, and Impex were selected only by the Chinese Style Association. The reasons for this obvious gap are as follows: First, the fitness equipment industry is accustomed to working with traditional mechanical methods. Sales of light industrial equipment are only satisfied with "profit effect" marketing, but lack of emotion, desire, mentality, and humanistic creative spirit. Secondly, due to the small size of the enterprises, the destruction of each other and the lack of competition, etc., the mutual reciprocity cannot be produced. Third, market development is relatively backward. According to expert studies, it will take 20 to 30 years to change people's living standards including food and clothing in developed countries[1]. Chinese food and clothing have been solved in the last 30 years, and the market still needs time to grow. Therefore, the development of fitness equipment also requires a lot of work to do.

Combining the theory of ergonomics, the design of this new rehabilitation exercise machine is to simulate skiing, jogging and exercise bikes, which has good practicality and low cost. The fitness equipment designed in this study is very suitable for rehabilitation exercises for people with lower limb disabilities.

\section{MATERIAL AND METHOD}

\subsection{The ergonomic theory}

Ergonomics is a comprehensive and interdisciplinary discipline, it covers the disciplines of physiology, psychology, biomechanics, surveying, human anatomy, environmental protection, engineering, management, architecture and lighting engineering, chromatics and informatics [2-5]. While ergonomics integrates the above disciplines, it takes human factors into consideration and realizes the overall design of human-machine, which provides a more comprehensive theoretical basis for designing products that integrate with humans. In this study, the rehabilitation fitness machine is a rehabilitation training machine designed based on ergonomic theory.

The study of human body parameters in ergonomics is the efficiency of cooperation between human and object under the action of space[6-8]. With the development of modern industry, the adaptation of humans to machines has gradually transformed into the 
adaptation of machines to humans. The parameters of humans have played a positive role in promoting the development of product design[9-12]. To sum up, it can be summarized into the following four aspects:

1) It can provide systematic human body parameter data for product design.

2) It can provide scientific basis for the rationality of product function and form.

3) It can provide design criteria for environmental factor variables of product design.

4) It can provide more comprehensive theoretical support for the systematic design of products.

Dimension measurement is the most basic problem in the product design process. It makes the designed product more consistent with human physiological characteristics, and makes people more comfortable in the space when the product is used. The dimensional measurement which involved in ergonomics is divided into functional dimensions and anthropometric dimensions. The initial size will depend on the experience of engineers and designers to develop the relevant size of the product
$[13,14]$. With the progress of society and the improvement of living standards, the measurement of human body size is gradually accepted, and corresponding data collection and measurement standards have been established. National standards provide some basic anthropometric measurement standards. After various fitness equipment are designed, relevant data should be obtained according to the standards $[15,16]$.

In this study, the human body parameter data is obtained by the photographic method, there are three main steps: marking the measurement object, selecting the reference position and shooting angle, and detecting the pixel distance using image processing software. Therefore, the actual size of the measurement object is equal to the ratio of the pixel measurement length and pixel distance of the base actual length.

The experimental equipment required for the measurement are as follows: a camera with 16 megapixel, a tripod, a seat, a pole, and the camera is used to capture images of the front of the person in the standing position and the side of the sitting position. The measurement results are shown in Table 1.

TABLE I. STATISTICAL TABLE OF HUMAN BODY

\begin{tabular}{cccc}
\hline Measuring project & $\begin{array}{c}\text { The national } \\
\text { standard(mm) }\end{array}$ & $\begin{array}{c}\text { The average } \\
\text { data }(\mathrm{mm})\end{array}$ & The difference(mm) \\
\hline Hand length & 183 & 193 & 10 \\
Height & 1678 & 1754 & 76 \\
Upper arm length & 313 & 273 & 40 \\
Forearm length & 237 & 262 & 25 \\
Chest width & 280 & 301 & 21 \\
Foot width & 96 & 104 & 8 \\
Foot length & 247 & 275 & 28 \\
Shoulder width & 375 & 401 & 26 \\
\hline
\end{tabular}

\subsection{The space design}

The operating space refers to the total space required to operate the machine and the space occupied by the processing object. The space required for machinery, equipment and tools should be reasonably arranged in accordance with the operating requirements of a reasonable space arrangement. Since the machine is operated manually, the comfortable and convenient work conditions should be created. Therefore, the operating device and display device should be reasonably arranged according to the needs of the operator. The excellent working space enables the operator to work safely, reliably, comfortably, and conveniently, which helps improve work efficiency. The design of the working space should be people-oriented, and on the basis of providing a working environment for the operator, create a safe, comfortable, economical and efficient operating space.

The work area is the area that can be reached by the operator's hands and feet, and the optimal work area must be calculated. The traditional research methods of ergonomics have proposed a horizontal operation field and a vertical operation field through a large number of experiments, and provided suitable operation fields on a single plane. When determining the operating range of the hand, the relevant parts and equipment must be arranged within the daily working range, so that comfortable operation will improve work efficiency.

\subsection{The display device design}

The display device can provide people with fast, convenient and accurate visual information, so the layout of the display device should be reasonably arranged according to human visual characteristics to improve reading efficiency and accuracy. In daily life and work, some experience have received by us, when an object is observed, the object in the plane to the eyes is of different angles, the effect is different, one of the reasons is the best viewing angle of the problem, as shown in Figure 1. 


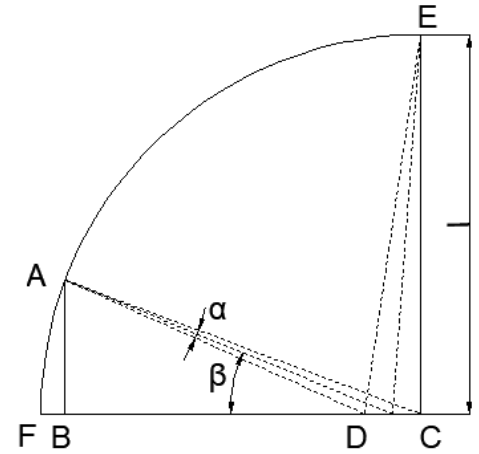

Figure 1. observation way and the best observation Angle $\beta$ is the line of sight $\mathrm{AC}$ for in horizontal angle with $\alpha$, 1 was distance, the geometric relationships in the figure is available:

$$
\alpha=\arccos \frac{4 l^{2}-a^{2}}{\sqrt{16 l^{4}+a^{4}-8 a^{2} l^{2} \cos 2 \beta}}
$$

In the above formula, $\alpha$ is the sight angle, it is seen from the formula, angle $\alpha$ is a function of the observation angle of $\beta$, if $\mathrm{l}=560 \mathrm{~mm}, \mathrm{a}=5 \mathrm{~mm}$, so $\alpha$ is changing with $\beta$.

TABLE II. $\alpha$ CHANGING WITH $\beta$

\begin{tabular}{|c|c|c|c|c|c|c|c|c|c|c|c|}
\hline viewing angle $\beta$ & 0 & 10 & 20 & 30 & 40 & 50 & 60 & 70 & 75 & 80 & 90 \\
\hline visual angle $\alpha$ & 0 & 0.089 & 0.175 & 0.256 & 0.329 & 0.392 & 0.443 & 0.481 & 0.494 & 0.504 & 0.511 \\
\hline
\end{tabular}

As shown in Table 2, with the increase of observation angle, angle $\beta$ increases, when $\beta=90^{\circ}$, the sight is vertical, $\alpha$ reach maximum, it is fixed when $\beta$ change, $\alpha$ is become bigger, it is showed that $\alpha$ in eye retina is become bigger, target detail ability is stronger, increase observation is more precision. Therefore, when a visual display unit is decorated, the surface of display the information should be perpendicular to the sight of the observer as much as possible to ensure the highest observation precision. If the condition can not reach, the display surface can also reach $70^{\circ}$ to $80^{\circ}$.

\subsection{The structure of the mechanical components design}

In the study, a six-link mechanism is used to design a fitness rehabilitation machine in space, and performs simple exercise training with two pedals. The principle of the six-link mechanism is consistent with ergonomics and is more humane, as shown in Figure 2. Ankle motion principle is adopted when a people is in walking, brisk walking or running, the movement of the foot pedals at this track can guide the user, the fitness machine action is consistent with the natural pace during the entire exercise. The foot does not completely leave the foot pedal, the knee is not impacted during the exercise. When the exerciser steps on the foot, he starts muscle movements at the same time, folding movement and coordination are performed during the arms of push-pull process, the legs, arms, chest and back are fully and comprehensively exercised.

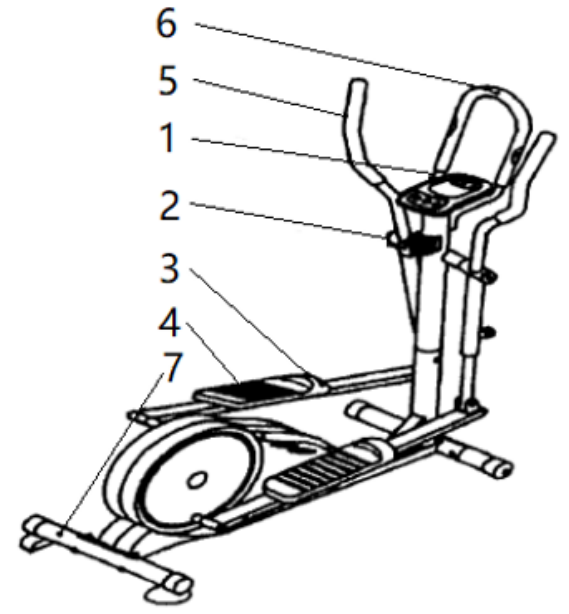

1 - display device; 2 - damping; 3 - pedal flange; 4 - foot pedal; 5 - activity armrest; 6 - fixed armrest; 7 - chassis

Figure 2. The main structure of the rehabilitation exercise machine

The rehabilitation fitness machine mainly includes the user, the machine body and the data statistics system. In the whole rehabilitation fitness machine system, human is the main body of the whole system design, and it is also the core part. Users use machines to achieve their fitness goals, and the machines record and respond accordingly through user input instructions, and then complete various commands. By recording data, the machine can monitor the usage in real time and achieve equipment maintenance. The following is a schematic diagram of the structure of the rehabilitation fitness machine fitness system, as shown in Figure 3. 


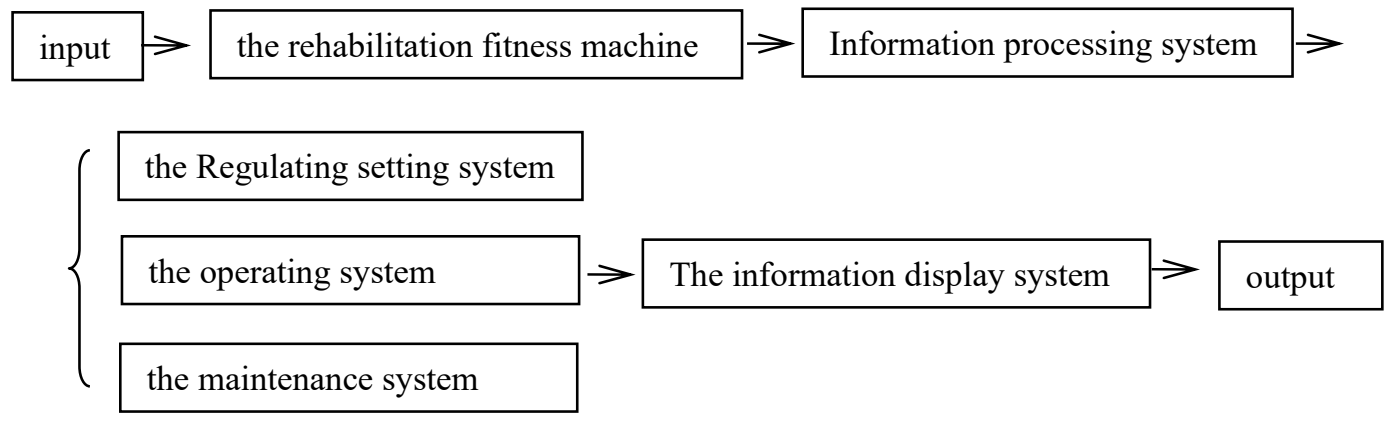

Figure 3. The structure of the rehabilitation fitness machine system

The structure of the rehabilitation fitness machine determines the functions to be achieved by the fitness machine, and its structural components mainly included: the system host, the operation system of the twist waist disk, the pedal operation system, the maintenance system, as shown in Figure 4.

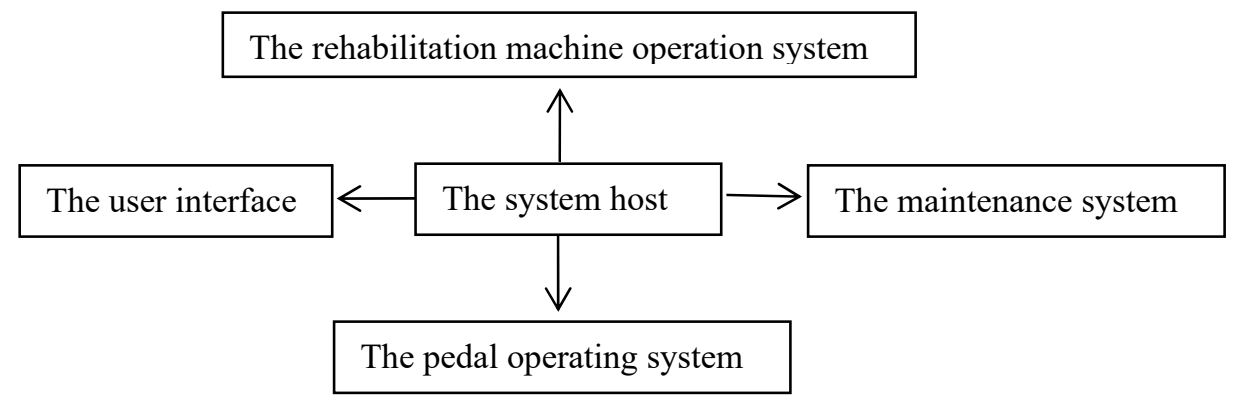

Figure 4. The composition of the rehabilitation fitness machine system

\subsection{The maintenance of rehabilitation fitness machine}

Equipment maintenance is an important means to ensure the normal operation of equipment in the process of using objective requirements. Due to the high frequency of use of the fitness machine, daily maintenance is very important to extend its service life. After using the fitness machine, lubricate joints should be applied to the mechanism to reduce frictional resistance between each other. Parts should always be cleaned with a clean cloth to maintain the normal performance of each part. If one or two drops of oil are regularly used on roller bearings, rolling bearings can ensure better performance. The screws of each machine should be checked regularly, if the bolts and nuts are loose, they should be locked immediately with a tool.

\section{RESULTS AND DISCUSSION}

After the market demand and existing fitness rehabilitation equipment are analyzed, a set of suitable design scheme of rehabilitation fitness machine for the disabilities is proposed, and the design concept is introduced in detail. According to the requirements of patients with waist, neck and shoulder diseases, this article uses the basic principles and methods of ergonomics to design the rehabilitation machine. Through the study of anthropometrics, psychology and human biomechanics, combined with the applicability of product design, the function of the fitness machine and the rationality of the design were verified.The conclusions are as follow:

1) The design scheme is feasible, the exercise intensity is also large, and it is suitable for almost everyone to use, and the fitness effect is obvious.

2) The design is similar to the exercise bike, and its main function is to exercise leg muscles, which is both energy saving and environmentally friendly.

3) The design meets the conditions of strength and stability, and the rehabilitation fitness machine is safe and reliable.

\section{ACKNOWLEDGMENTS}

The authors acknowledge that this research was financially supported by National Natural Science Foundation of China (51905387).

\section{REFERENCE}

1. Colin G. D. Service, quality and human factors. AI\&Society, 2003, 17(2): 78-96.

2. Chris B., James K., Haniff D. Ergonomics of wearable computers. Mobile Networks and Applications, 1999, 4(1): 15-21.

3. Berguer R., Forkey D.L., Smith W. D. Ergonomic problems associated with Iaparoscopic surgery. Surgical Endoscopy. 1999, 13(5): 466-468.

4. Paula C. B. Efficacy of Office Ergonomics Education. Journal of Occupational Rehabilitation. 2000, 10(4): 243-255. 
5. John R. Virtual environments applications and applied ergonomics. Applied Ergonomics. 2003, 1(3) 3-9.

6. Singleton W. T. The Body at Work-Biological Ergonomics.l st Edition. London, UK. 2002.

7. By D., Oborne J., Boarer P.A. Subjective response to whole-body vibration. The effects of posture Ergonomics, 1982, 25: 673-681.

8. Park M. Y. Ergonomic design and evaluation of a new VDT workstation chair with keyboard-mouse support. International Journal of Industrial Ergonomics. 2001, 26(5): 537-548.

9. Narelle S. A case study of the ergonomics information in aheavy engineering design process. International Journal of Industrial Ergonomics. 2001, 26(3): 417-424.

10. Meister D. Behavioral analysis and measurement methods, New York, wiley. 2005.
11. Meister D. Human factors: Theory and practise. New York, Woley,2005, 10(3):201-215.

12. William R. Development and evaluation of the Maintenance Error Decision Aid (MEDA) process. International Journal of Industrial Ergonomics. 2001, 26(2): 261-276.

13. Lssachar G. Muscular effort on four keyboard designs. International Journal of Industrial Ergonomics. 2001, 26(1): 109-112.

14. Chen Y. I. Changes in lifting dynamics after localized arm fatigue. International Journal of Industrial Ergonomics. 2001, 25(6): 597-609.

15. Stephenson J,Bauman,Armstrong T. The costs of illness attributable to physical inactivity in Australia[M], Canberra: Commonwealth department of health and aged care, 2006, 10(2),210-218.

16. Jacob Mack, Sarah Stojsih, Don sherman.Amateur boxer biomechanics and Punch Force.[J].Annals of Biomedical Engineering, 2005, 6(2):115-119. 